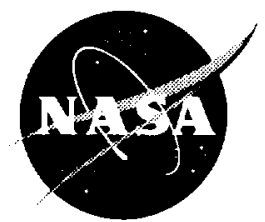

\title{
Numerical Speed of Sound and its Application to Schemes for all Speeds
}

Meng-Sing Liou

Glenn Research Center, Cleveland, Ohio

Jack R. Edwards

North Carolina State University, Raleigh, North Carolina

Prepared for the

14th Computational Fluid Dynamics Conference

sponsored by the American Institute of Aeronautics and Astronautics

Norfolk, Virginia, June 28-July 1, 1999

National Aeronautics and

Space Administration

Glenn Research Center 


\section{Acknowledgments}

The first author thanks Pieter Buning of Langley Research Center and William Chan of Ames Research Center, who have provided consultation concerning the OVERFLOW code and its utility tools. This study was sponsored by the Turbomachinery and Combustion Technology project, managed by Kaz Civinskas of Glenn Research Center.

Available from

NASA Center for Aerospace Information

7121 Standard Drive

Hanover, MD 21076

Price Code: A03
National Technical Information Service 5285 Port Royal Road Springfield, VA 22100 Price Code: A03 


\title{
NUMERICAL SPEED OF SOUND AND ITS APPLICATION TO SCHEMES FOR ALL SPEEDS
}

\author{
Meng-Sing Liou* \\ NASA Glenn Research ('enter at Lewis Field, MS 5-11, Cleveland. OH 44135, USA \\ fsmsl gyinyan.grc.nasa.gor \\ Jack R. Edwards $\}$ \\ North Carolina State Iniversity, Raleigh, NC 27695, USA \\ edwards $\mathrm{q}$ animate.mae.ncsu.edu
}

\begin{abstract}
The concept of "numerical speed of sound" is proposed in the construction of numerical flux. It is shown that this variable is responsible for the accurate resolution of discontinuities, such as contacts and shocks. Moreover, this concept can be readily extended to deal with low speed and multiphase flows. As a result. the numerical dissipation for low speed flows is scaled with the local fluid speed, rather than the sound speed. Hence, the accuracy is enhanced. the correct solution recovered. and the convergence rate improved. We also emphasize the role of mass flux and analyze the behavior of this flux. Study of mass flux is important because the numerical diffusivity introduced in it can be identified. In addition, it is the term common to all conservation equations.

We show calculated results for a wide variety of flows to validate the effectiveness of using the numerical speed of sound concept in constructing the numerical flux. We especially aim at achieving these two goals: (1) improving accuracy and (2) gaining convergence rates for all speed ranges. We fiud that while the performance at high speed range is maintained, the flux now has the capability

*Assocjate Fellow, AIAA

SSenior Member. AIAA

Copyright 1998 by the American Institute of Aeronautics and Astronautics. Inc. No copyright is asserted in the United States under Title 17. U.S. Code. The U.S. Government has a royalty-free license to exercise all rights under the copyright claimed herein for Governmental Purposes. All other rights are reserved by the copyright owner.
\end{abstract}

of performing well even with the low speed flows. Thanks to the new numerical speed of sound. the convergence is even enlanced for the flows outside of the low speed range. To realize the usefulness of the proposed method in engineering problems. we have also performed calculations for complex 3I) t urbulent flows and the results are in excellent agreement with data.

\section{Introduction}

Numerical representation of inviscid fluxes. namely the numerical flux function. has been a subject of intensive effort by many researchers during the last three decades. Despite the enormous progress that has been achieved, further analysis and deeper nuderstanding into these numerical procedures continue to draw interest and the findings are being reported. In this paper we will introduce the concept of the numerical spect of sound. This concept turns out to be very useful in designing a numerical flux in order to meet certain criteria. Moreover, as will be seen later, the numerical speed of sound lends itself nicely to the formulation of numerical schemes for all speeds. In other words. it 
plays an important role in bridging the gap between discretization schemes suitable for incompressible flows and those suitable for compressible flows.

For illustrative purposes. we shall begin by cousidering the 1D flux for ideal gas. The inviscid numerical flux function is written as a sum of convective and pressure fluxes:

$$
\mathbf{f}=\rho u\left(\begin{array}{c}
1 \\
u \\
H
\end{array}\right)+\left(\begin{array}{l}
0 \\
p \\
0
\end{array}\right)=\dot{m}\left(\begin{array}{l}
1 \\
u \\
H
\end{array}\right)+\left(\begin{array}{l}
0 \\
p \\
0
\end{array}\right)
$$

It is noted that a common mass flux $i$ appears in all equations. This is also true for multidimensions. Since the mass flux is common for all equations. its effects will thus perpetuate in all variables. Hence, we suggest that it is desirable to observe this fact at the discrete level when derising a new scheme. However. this fact is not entirely enforced in several modern numerical schemes.

The AUSM-family schemes writes the intterface flux $f_{1 / 2}$, in a form mimicking the conttinum flux, Eq. (1). as

$$
\mathbf{f}_{1 / 2}=\dot{m}_{1 / 2}\left(\begin{array}{l}
1 \\
u \\
H
\end{array}\right)_{j / j+1}+\left(\begin{array}{c}
0 \\
p_{1 / 2} \\
0
\end{array}\right)
$$

where the cell interface straddles the cells $j$ and $j+1$ and the subscript $j / j+1$ denotes that the vector $(1 . u . H)$ is evaluated with either $j$ or $j+1$ values according to whether $\dot{m}_{1 / 2}$ is positive or negative. The detailed description of $\dot{m}_{1 / 2}$ and $p_{1 / 2}$ can be found in $[2-1]$.

\section{Numerical Speed of Sound}

To understand the idea of mumerical speed of sound. we first consider the celebrated Van Leer scheme [1], which turns out to be an important foundation for the development of recent schemes such as the AUSMfamily [2-4]. The mass flux of the Van Leertype scheme is,

$$
\dot{m}_{1 / 2}=\rho_{j} a_{j} \mathcal{M}_{(4, \beta)}^{+}\left(M_{j}\right)+\rho_{j+1} a_{j+1} \mathcal{M}_{(4, \beta)}^{-}\left(M_{j+1}\right) \text {. }
$$

where $\mathcal{M}_{(4,3)}^{ \pm}$are the split Mach number functions defined by the 4th-degree (or second degree in [1]) polynomials in the subsonic range:

$$
\mathcal{M}_{(1,3)}^{ \pm}(M)=\left\{\begin{array}{l}
\mathcal{M}_{(1)}^{ \pm}, \text {if }|M| \geq 1 . \\
\mathcal{M}_{(2)}^{ \pm}(M)\left[1 \mp 16,3 \mathcal{M}_{(2)}^{\mp}(M)\right] \\
\text { otherwise, }
\end{array}\right.
$$

where

$$
\mathcal{M}_{(1)}^{ \pm}=\frac{1}{2}(M \pm|M|)
$$

and

$$
\mathcal{M}_{(2)}^{ \pm}= \pm \frac{1}{4}(M \pm 1)^{2} \cdot \S
$$

Other forms are possible. but are not essential in the discussion in this paper. The scheme. Eq. (3). is known to produce a dissipative result at a contact discontinuity. As $M=M_{j}=M_{.+1}=0$. the mass flux does not vanish.

$$
\dot{m}_{1 / 2}=\left(\rho_{j} a_{j}-\rho_{j+1} a_{j+1}\right) / 4
$$

It is clear in the Van Leer scheme that two variables, namely $(\rho, a)$. need to be dealt with if a mass flux is to vanish at $M=0$. It is due to this observation that a common speed of sound $a_{1 / 2}$ is introduced in the AISMM+ and AUSMDV schemes. Let's modify Eq. (3) by using $a_{1 / 2}$.

$$
\dot{m}_{1 / 2}=a_{1 / 2}\left(\rho_{j} \mathcal{H}_{(4,3)}^{+}\left(M_{j}\right)+\rho_{j+1} \mathcal{H}_{(1, j)}^{-}\left(M_{j+1}\right)\right) .
$$

where the exact definition of $a_{1 / 2}$ is not important for the moment. but is certainly the central topic of this paper. The limiting form of $\dot{m}_{1 / 2}$ at $M=0$ becomes.

$$
\dot{m}_{1 / 2}=a_{1 / 2}\left(\rho_{j}-\rho_{j+1}\right) / 1 \neq 0 .
$$

$\S$ The coefficient in (19h) of JCP 129, 364-382 (1996). should be $1 / 4$, not $1 / 2$. 
Clearly the common speed of sound $a_{1 / 2}$ alone is not enough to make the mass flux vanish at $M=0$, and we need something extra. A novel approach is proposed in the AltsMDV scheme in which the split Mach number functions $\mathcal{M}_{(1)}^{ \pm}$are addled as an anti-diffusive mechanism to fully cancel the excessive dissipation. The interested reader is referred to [4] for cletails.

In a different approach. AUS.M ${ }^{+}$defines the mass flux as

$$
\dot{m}_{1 / 2}=\frac{a_{1 / 2}}{2}\left[M_{1 / 2}\left(\rho_{j}+\rho_{j+1}\right)-\left|M_{1 / 2}\right|\left(\rho_{j+1}-\rho_{j}\right)\right] .
$$

where

$$
M_{1 / 2}=\mathcal{M}_{(4,3)}^{+}\left(M_{j}\right)+\mathcal{M}_{(4,3)}^{-}\left(M_{j+1}\right) .
$$

Since $M_{1 / 2}=0$ when $M_{j}=M_{j+1}=0$, we automatically get $\dot{m}_{1 / 2}=0$.
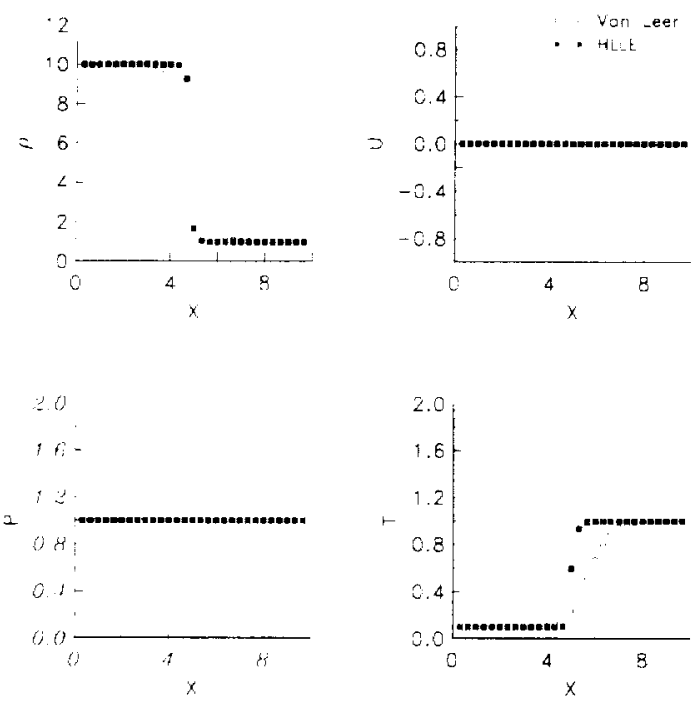

Fig. 1 Stationary contact discontinuity by the Van Leer and HLLE schemes

We remark that both AUSMDV and $\mathrm{Al}^{-} \mathrm{SM}^{+}$yicld the desired result for a stationary contact discontinuity of any strength in $\left(\rho_{\gamma}, \rho_{j+1}\right)$ with an arbitrary $a_{1 / 2}$. Figure 1 displays the solution at $t>0$ for a stationary contact discontinuity. It is seen that the two dissipative schemes. Van Leer and HLLE [5]. will destroy the discontinuity at the first time step: Van Leer flux is seen to be more dissipative llan the HLLE flux.

While a stationary contact can be exactly captured by the original AUSM scheme [2]. failure is encountered in the case of moving contact discontinuity. as shown in Fig. 2. This is also the reason for introducing a common speed of sound in the AUSM scheme to achieve this and other improvements, thus leading to the improved scheme called $\mathrm{AISM}^{+}[3]$. Since the common speed of sound is introduced as a means to yield an accurate solution, which is only meaningful in the numerical framework, hence it is hereafter termed "numerical" speed of sound.

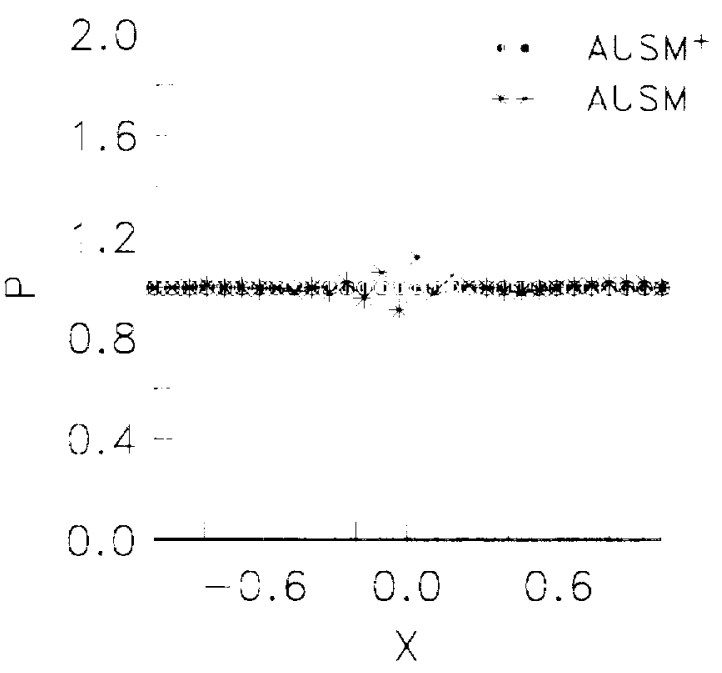

Fig. 2 Slowly moving contact: comparison of ACSM and ACSM+ solutions.

As stated earlier. the contact (stationary and moving) discontinuities can be accurately resolved with an arbitrary numerical speed of sound $a_{1 / 2}$. This leaves us one degree of freedom to determine $a_{1 / 2}$ so that another interesting property can be met. for example. exact capturing of a stationary shock discontinuity. This is accomplished in the ATSMfamily schemes by setting (detailed deriva- 
tion can be found in [3]).

$$
a_{1 / 2}=\min \left(\grave{a}_{L}, \dot{a}_{R}\right), \quad \grave{a}=a^{* 2} / \max \left(a^{*},|u|\right) .
$$

where $a^{*}$ is the critical speed of sound. For ideal gas, we have

$$
a^{* 2}=\frac{2(\gamma-1) H}{\gamma+1} .
$$
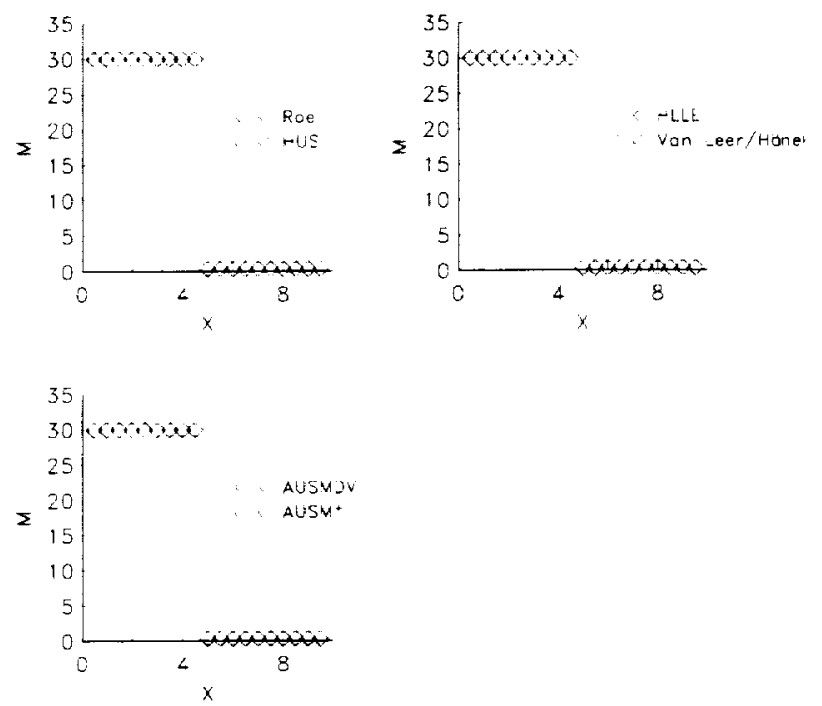

Fig. 3 Stationary shock discontinuity: a numerical speed of sound is inserted in each of the schemes included.

It is well known that a single shock can be captured by the Roe scheme with the socalled Roe averaged quantities. One of these quantities is the averaged speed of sound. which also is "numerical" in nature. The idea of regarding the quantity $a_{1 / 2}$ as a free variable turns out to be rather powerful in the sense that the idea can be applied to other schemes equally well. Firstly, the two intermediate shock points by the Van Leer scheme. including its original form [1] and Hänel's modification [6] for the energy flux. are now dramatically removed by simply incorporating this special numerical speed of sound. Secondly, the HLLE schemes can be made to satisfy the same property by using the formula in Eq. (12) as well. Thirdly, in addition to the familiar Roe arraged speed of sound, we find that

$$
a_{1 / 2}=\max \left(\grave{a}_{L}, \grave{a}_{R}\right)
$$

instead of "min" used in Eq. (12), can also yield an exact shock profile. The result in Fig. 3 for the Roe scheme was obtained with this formula. To our knowledge, these choices of $a_{1 / 2}$ to achieve the exact property with the HLLE and Roe schemes are previously unknown in the literature.

\section{Formulation for All Speeds}

It is widely known that the standard form of compressible equations, discretized with either centered or upwind schemes. vields two major effects on the solution as the flow speed approaches zero: (1) a drastic slowdown or level-off of convergence rate, (2) an inaccurate or even incorrect solution. An effective way of dealing with the first point is by inserting a premultiplying matrix to the timederivative term, thus it is usually called the local preconditioning technique. Many authors, notably the schools of Merkle [7]. Van Leer [s]. and Turke] [9]. have made significant contributions in this area. For the second point, the inaccuracies in the upwind schemes are primarily due to the incorrect scaling of the dissipation term as $\boldsymbol{M} \rightarrow 0$. In fact, the dissipation turns out to be scaled by the sound speed at low Mach numbers. thus yielding excessive numerical dissipation. This immediately suggests that numerical fluxes need to be modified to correct this situation.

The preconditioning is done to essentially alter the eigenvalues of the hyperbolic systems so that the wave speeds become more or less equalized. To see this. we define a 
condition number $k$ as the largest ratio of eigenvalues,

$\kappa=\frac{|u|+a}{|u|} \rightarrow \infty$, as $|u| \rightarrow 0$, and a held fixed

Clearly there is a large disparity of wave speeds and as a result this has been identified as the source of slow (or no) convergence as $|u| \rightarrow 0$. We bring the attention that the limiting taken in Eq. (15) is fundamentally different from that by holding $u$ fixed and taking $a \rightarrow \infty$. The former is more general, allowing low speed in a compressible medium (where $a$ is finite), and it is especially useful for dealing with situations with disparate speeds of sound in the flow.

Now if the numerical system is modified in such a way that it has a corresponding speed of sound, $\dot{a}$. which behaves like $|u|$ as it approaches to zero. then the condition number will remain order of unity. That is,

$\kappa=\frac{|u|+\grave{a}}{|u|} \rightarrow O(1)$. while $\grave{a} \rightarrow 0$. as $|u| \rightarrow 0$.

Hence. condition number remains of order unity at low speeds. The numerical dissipation based on this new speed of sound now scales with the local speed $|u|$. instead of local speed of sound $a$. To aclijeve this goal. the trick is to manipulate the hyperbolic srstem with the premultiplying matrix. Therefore. the system is re-scaled. The numerical speed of sound comes in handy for achieving the purpose. lising the time-clerivative premultiplying matrix proposed by Weiss and Smith [10], the time dependent governing (Euler or Navier-Stokes) equations are cast in the following form:

$$
\mathrm{\Gamma} \frac{\partial H}{\partial t}+\frac{\partial F}{\partial x}+\frac{\partial \sigma_{r}}{\partial y}=0
$$

where $I T$ is a vector of primitive variables. $(p, u, v, T)^{T}$ and all other variables are standard. The preconditioning matrix takes the form:

$$
\Gamma=\left[\begin{array}{cccc}
\Theta+\frac{1}{R T} & 0 & 0 & -\frac{\rho}{T} \\
u\left(\Theta+\frac{1}{R T}\right) & \rho & 0 & -\frac{\rho u}{T} \\
\nu\left(\Theta+\frac{1}{R T}\right) & 0 & \rho & -\frac{\rho r}{T} \\
H\left(\Theta+\frac{1}{R T}\right)-1 & \rho u & \rho r & \rho\left(C_{p}-\frac{H}{T}\right)
\end{array}\right]
$$

where

$$
\begin{gathered}
\Theta=\frac{1}{a^{2}}\left(\frac{1}{M_{*}^{2}}-1\right) . \\
M_{*}^{2}=\min \left(1, \max \left(M^{2}, M_{\omega}^{2}\right)\right) .
\end{gathered}
$$

The cutoff parameter $M_{c}$, is introduced to prevent a singularity at stagnation point. It is a user-specified parameter. Infortunately it does have some effects on the solution in some situations, as will be displayed later (in Fig. 4). The effect of $\boldsymbol{M}_{\text {to }}$ generally is minor. but could be of significance in some situations. A pressure difference term. as suggested by Wriss [11]. can be added to enhance robustness near a stagnation point. The reference quantity $M_{*}^{2}$ is also bounded from above to unity as local $M$ exceeds one. As a result the eigenvalues of the flux Jacobian of $F$ with respect to $\mathrm{H}^{\circ}$. i.e. $\mathrm{I}^{-1} \partial \mathrm{F} / \partial \mathrm{J}$ are 1. and

$u^{\prime} \pm a^{\prime}=\frac{1+M_{*}^{2}}{2}\left(u \pm a \frac{\sqrt{\left(1-M_{*}^{2}\right)^{2} M^{2}+4 M_{*}^{2}}}{1+M_{*}^{2}}\right)$,

where $M=u / a$ is the unscaled Mach number. Several remarks can be made concerning the eigenvalues of the preconditioned system. Firstly, we have a bound for the coefficient.

$$
\frac{1}{2}<\frac{1+M_{*}^{2}}{2} \leq 1
$$

Secondly, the speed of sound is now re-scaled by the scaling factor $f\left(M ; M_{*}\right)$. Thus, a new speed of sound can be defined.

$$
i=f\left(M ; M_{\times}\right) a .
$$

$$
f\left(M: M_{*}\right)=\frac{\sqrt{\left(1-M_{*}^{2}\right)^{2} M^{2}+4 M_{*}^{2}}}{1+M_{*}^{2}} .
$$


It is reminded that a in the above equation be given in $\mathrm{Eq}$. (12). The scaling factor is also bounded.

$$
1 \geq f \geq \begin{cases}|M| & \text { if } 1>>M^{2}>>M_{w}^{2} \\ 2 M_{w} & \text { if } 1>>M_{w}^{2}>>M^{2} .\end{cases}
$$

That is, the scaling factor $f$ is bounded from below by the larger of the local and cut-off Mach numbers. ('learly, the variable $\dot{u}$, introduced as above. has been utilized for nothing but numerical purposes. Hence it fits in the spirit of numerical speed of sound.

Now equipped with the newly defined numerical speed of sound, we can readily incorporate it into the formulation of the AISMfamily schemes. In this paper, we will illust rate the concept within the framework of $\mathrm{NSSM}^{+}$only: similar procedures can be also implemented in the other schemes. Let us use the above scaling factor to define the numerical speed of sound, $\grave{a}_{1 / 2}=\dot{a}\left(\mathbf{U}_{j} \cdot \mathbf{U}_{j+1}\right)$. at the interface $1 / 2$. The mass flux of the $\mathrm{MSSM}^{+}$scheme in Eq. (10) now can be recast by inserting this numerical speed of sound.

$$
\dot{m}_{1 / 2}=\frac{i_{1 / 2}}{2}\left[M_{1 / 2}\left(\rho_{j}+\rho_{j+1}\right)+\left|M_{1 / 2}\right|\left(\rho_{j}-\rho_{,+1}\right)\right] .
$$

where the interface Mach number is given by,

$$
M_{1 / 2}=\mathcal{M}_{(4,3)}^{+}\left(\bar{M}_{j}\right)+\mathcal{M}_{(4,3)}^{-}\left(\bar{M}_{j+1}\right) .
$$

and the arguments in $\mathcal{U}_{(A, j)}^{ \pm}$have been substituted with

$$
\begin{aligned}
& M_{j}=\frac{1}{2}\left[\left(1+M_{*}^{2}\right) \dot{M}_{j}+\left(1-M_{*}^{2}\right) \dot{M}_{j+1}\right] . \\
& M_{j+1}=\frac{1}{2}\left[\left(1+M_{*}^{2}\right) \dot{M}_{j+1}+\left(1-M_{*}^{2}\right) \dot{M}_{j}\right] .
\end{aligned}
$$

The tilde Nach numbers, $\left(\dot{M}_{j}, \dot{i}_{j+1}\right)$. are now defined with respect to the numerical speed of sound $a$, i.e.

$$
\grave{i}=\frac{u}{a} .
$$

This is the scaled Mach number. which will revert to the local physical Mach number at supersonic speeds. The step taken in Eqs. (28)-(29) is necessary to eliminate unphysical numerical diffusion present in the pressure flux splitting and to enhance the robustness. Test cases have shown that the definitions in Eqs. (28)-(29) are not strictly necessary for the mass flux definition; the simpler alternatives $\bar{M}_{j}=\dot{M}_{j}$ and $\bar{M}_{j+1}=\dot{M}_{j+1}$ can be used as well. These result in a slightly more dissipative scheme for low Mach number calculations.

For identification purpose, we now call this extended method, $\mathrm{AUSM}^{+}$a, to highlight the role of the numerical speed of sound a.

In Fig. 4. we demonstrate that the numerical dissipation is greatly reduced even if the scaled numerical sound speed is included in the Van Leer scheme for solving the contact problem. The error will begin to reach the end of the computation domain in 1.5 steps with the original scheme, but still remains inside after 180 steps (not shown in the figure) with the scaled numerical sound speed. Hence. confirming a much smaller dissipation - as evident in Fig. 4.
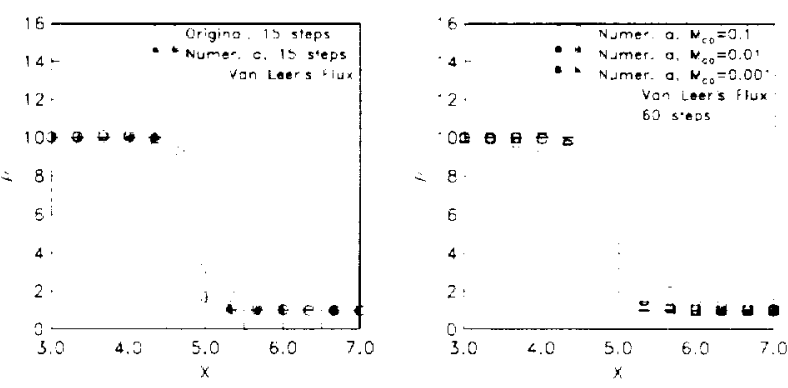

Fig. + Stationary contact discontinuity showing the effectiveness of using the scaled numerical speed of sound in the Van Leer Flux.

Another interesting result is also revealed in Fig. 4. The plot on the right demonstrates 
the dissipative effect of the cutoff Mach number $M_{c o}$ on the spreading of the stationary contact. The smaller the $M_{c o}$ is set. the sharper the contact discontinuity gets results being closer to the exact solution denoted by solid line, indicating that the numerical dissipation increases with the value of $M_{c o}$.

To further improve the convergence in the low speed range, it is found beneficial in Edwards and Liou [12] that a pressure diffusion term $\dot{m}_{p}$ be included in the mass flux.

$$
\dot{m}_{1 / 2}=\mathrm{Eq} \cdot(26)+\dot{m}_{p} .
$$

We can write $\dot{m}_{p}$ in the following general form.

$$
\dot{m}_{p}=\frac{\dot{\partial}_{1 / 2}}{\mathcal{D}}\left(1-M_{*}^{2}\right) \Delta \cdot \mathcal{M}\left(p_{i}-p_{j+1}\right)
$$

where

$$
\begin{aligned}
& \Delta . \mathcal{M}=\left[\mathcal{M}_{(4, j)}^{+}\left(M_{j}\right)-\mathcal{M}_{(1)}^{+}\left(M_{j}\right)\right. \\
& \left.-\mathcal{M}_{(4,3)}^{-}\left(M_{j+1}\right)+\mathcal{M}_{(1)}^{-}\left(M_{j+1}\right)\right] .
\end{aligned}
$$

and the function $\mathcal{D}$ in the denominator can take several forms. Based on the mass flux of the AUSMDV scheme, Edwards and Liou $[12]$ derived the pressure diffusion term for low Mach numbers,

$$
\mathcal{D}=M_{*}^{2}\left[\frac{p_{j}}{\rho_{i}}+\frac{p_{j+1}}{\rho_{.+1}}\right] .
$$

In this paper we suggest another formula,

$$
\begin{array}{r}
\mathcal{D}=\frac{1}{\rho_{.} \rho_{j+1}}\left[M_{\times}^{2}\left(p_{j}+p_{j+1}\right)\left(\rho_{j}+\rho_{j+1}\right)\right. \\
\left.-\left(p_{j}-p_{j+1}\right)\left(\rho_{j}-\rho_{j+1}\right)\right] .
\end{array}
$$

This is obtained by also starting with the AUSMDV mass flux and by imposing a condition that its weighting factors be bounded (see Appendix A). Furthermore, the last term can be omitted to guarantee that $\mathcal{D}$ be positive.

$$
\mathcal{D}=\frac{1}{\rho_{j} \rho_{j+1}}\left[M_{*}^{2}\left(p_{j}+p_{j+1}\right)\left(\rho_{j}+\rho_{j+1}\right)\right] .
$$

which looks similar to the first expression.

To see how the numerical speed of sound derived for the preconditioned system affects the discretization. it is useful to examine the mass flux in the limit of an incompressible flow. where $\rho$ is constant and $a_{1 / 2}$ (but not $a_{1 / 2}$ ) approaches infinity. Ising the definitions in Eqs. (26)-(32) and taking the limit $M_{*}^{2}$ tending to zero in Eqs. (28)-(29), we find that

$$
\begin{aligned}
\dot{m}_{1 / 2}= & \rho u_{1 / 2}+\frac{\sqrt{u_{1 / 2}^{2}+4 V_{*}^{2}}}{I_{*}^{2}}\left(p_{i}-p_{i+1}\right) \\
& \times r_{4}\left[\frac{1}{4}\left(\left|M_{I}\right|-1\right)^{2}+\beta\left(M_{I}^{2}-1\right)^{2}\right] .
\end{aligned}
$$

where

$$
u_{1 / 2}=\frac{1}{2}\left(u_{j}+u_{j+1}\right)
$$

and

$$
M_{l}=\frac{u_{1 / 2}}{\sqrt{u_{1 / 2}^{2}+4 l_{*}^{2}}} .
$$

The reference velocity $V_{*}$ is defined analogously with Eq. (20):

$$
\Gamma_{*}^{2}=\max \left(|\vec{V}|^{2}, \Gamma_{i j}^{2}\right) .
$$

This mass flux formula involves only the velocity field, pressure, and a constant density and is similar to that utilized in incompressible-flow discretizations on nonstaggered grids. Note that the physical sound speed completely disappears from the formulation in the incompressible limit. Advective upwind influences are present in the momentum equations and the (decoupled) energy equation through the switching process shown in Eq. (2). but only the pressurediffusion term provides dissipation for the continuity equation.

Moreover, the idea of using $a$ can be readily applied to solve multiphase flow problems 
in which both the density and Mach numbers can vary drastically with phase changes. More interestingly, the flow can go supersonically even though the fluid speed is low, due to a steep decrease in speed of sound. The reader is referred to [13] in this conference.

In what follows we summarize the steps involved for adding the numerical speed of sound in the ALSM ${ }^{+}$. These steps, although motivated for low-Mach number flows. are also valid for high Mach-number ones. The modifications detailed above also work for the pressure flux splitting (at least for perfect gases). serving to remove unphysical sources of numerical dissipation in the momentum equations. [12] Further modifications are necessary for real fluid applications. and the reader is again referred to [1:3] for details.

1. Tse the numerical speed of sound $\grave{a}_{1 / 2}$ (2:3) to define $\dot{M}_{j}$ and $\dot{H}_{j+1}$ appearing in Eqs. (28)-(29).

2. Replace the left- and right-state Mach number definitions by $\bar{M}_{j}$ and $M_{j+1}$.

3. Construct the mass flux as usual by using as input the $\left(\bar{M}_{j}, \bar{M}_{j+1}\right)$ defined in the previous step.

t. If desired. add contribution from pressure diffusion by using Eq. (32).

5. ('omplete evaluation of other fluxes.

This is all there is to it, involving a kind of pre- and post-processing of the usual ACSMfamily schemes, steps $1-2$ and 4 respectively. It is a matter of adding only a few more lines to the original $\mathrm{AISM}^{+}$colle. It is believed that other low-diffusion hybrid schemes can also be extended in a similar manner. We shall denote the scheme with the pressure diffusion term ATSM ${ }^{+}$-ap.

We now make remarks on the preconditioning matrix I. Wo have used the MeissSmith $\Gamma$ to arrive at the scaling function
$f\left(M ; M_{*}\right)$ in Eq. (24). Other preconditioners $[8,9]$ can be used as well. The procedure for extension will be precisely the same since all one needs is the eigenvalues of the preconditioned hyperbolic system. Thus, a new numerical speed of sound $a$ can be expressed in terms of the scaling function $f\left(M: M_{*}\right)$. However, no significant effect on the solution is anticipated because all these preconditioners yield more or less the same behavior in the limits of $M \rightarrow 0$ and 1 . Inless at low speed ( say $M_{\infty}<0.3$ ), it was found in our calculations not necessary to include the preconditioning matrix in solving the governing equations. In otler words, the scaling function can be incorporated alone. as in Eqs. (26)(29), in the numerical flux and improvements in accuracy and convergence can be realized.

\section{Results and Discussion}

In this section, we will present $2 \mathrm{D}$ and $3 \mathrm{D}$ Navier-Stokes solutions for turbulent flows over various geometries. The scheme proposed in this paper was implemented in the OVERFLOW code supplied by Buning et al. [14]. The turbulence eddy viscosity in all cases presented herein was calculated according to the Spalart-Allmaras one equation model [15]. All of the results presented below were obtained using an implicit scheme. The LHS operator was approximated with the standard central difference scheme plus appropriate artificial damping terms. (even though the RHS residual operator was represented with an upwind scheme!), it was then further factored and diagonalized in each space dimension.

The flux in the RHS operator was constructed with a third-order accurate interpolation for the primitive variables. together with limiter used by Koren [19]. The cutoff Mach number in Eq. (20) is given by $M_{c o}^{2}=M_{x}^{2} / 4$. However, the OVERFLOW 
code has a parameter controlling the use of the preconditioning matrix $\Gamma$. We kept the default value to be $3 M_{\infty}^{2} \leq 1$ under which condition $\Gamma$ was activated.

In this paper we will demonstrate the effectiveness of using the numerical speed of sound in calculating flows at all speeds, specifically focusing on two issues: (1) convergence rate and (2) accuracy. We will show that the convergence rate is improved for the entire flow speed regime and the calculated solutions are in excellent agreement with data.

\section{Shuttle External Tank}

This is an axisymmetric Shuttle external tank geometry with a sharp nose and blunt base. downstream of which a significant separation zone is created, see Fig. 8. One of the grid lines conforms to the body and grows outward and a plane consists of $88 \times 60$ grid points. Shown also are the meshes clustered around some key regions. one of which is in the middle to resolve a tiny notch (not visible to the scale). The free stream Reynolds number was fixed at 10,000 . But a fully turbulent flow from the nose was assumed in order to pose a more stringent condition for the assessment of convergence behavior. We have tested conditions from low Mach, transonic, to supersonic flows. Several schemes were considered, consisting of the standard $\mathrm{ACSM}^{+}, \mathrm{AlSSM}^{+}-\mathrm{a}$, and $\mathrm{ALSM}^{+}-$ap. with and without the Weiss-Smith preconditioner. In all calculations for this problem. we made 200 steps for each of two coarser grids prior to the finest grid. on which 3000 more steps were continued unless noted otherwise.

Table 1: Summary of convergence behavior due to various schemes for the shuttle external tank. $R_{x_{x}}=10000$.

\begin{tabular}{|c|c|c|c|}
\hline$M_{\text {Х }}$ & Scheme & So Precond. & Precond. \\
\hline 0.01 & $\begin{array}{c}\mathrm{AUSM}^{+} \\
\mathrm{ACSM}^{+}-\mathrm{a} \\
\mathrm{AUSM}^{+}-\mathrm{a}+\dot{m}_{p}\end{array}$ & \begin{tabular}{c}
\multicolumn{1}{c}{} \\
No converg. \\
No converg.
\end{tabular} & $\begin{array}{c}\text { Diverg. } \\
\sqrt{ } \\
V\end{array}$ \\
\hline 0.80 & $\begin{array}{c}\text { AISMM }^{+} \\
\text {AISMM }^{+}-\text {a } \\
\text { AUSM }^{+}-a+\dot{m}_{p}\end{array}$ & $\begin{array}{l}\sqrt{ } \\
\sqrt{ } \\
V\end{array}$ & $\begin{array}{l}\mathrm{V} \\
\mathrm{V} \\
\mathrm{V}\end{array}$ \\
\hline$\cdot 2.00$ & $\begin{array}{c}\mathrm{AISM}^{+} \\
\mathrm{AUSM}^{+}-\mathrm{a} \\
\mathrm{AlSM}^{+}-\mathrm{a}+\dot{m}_{p}\end{array}$ & $\begin{array}{l}\mathrm{V} \\
\mathrm{V} \\
\mathrm{V}\end{array}$ & $\begin{array}{l}\sqrt{ } \\
\sqrt{ } \\
\sqrt{ }\end{array}$ \\
\hline
\end{tabular}

Table I summarizes the comvergence behavior of the above combinations. We observe the following: (1) For low Mach numbers (approximately $M_{x}<0.3$ ). it was found necessary to use the time-derivative preconditioner $\Gamma$ so that the numerical dissipations in both the implicit and explicit operators are compatibly scaled. Otherwise, the calculation ejther diverged or stagnated. (2) For flows at transonic speeds or higher. the time-derivative preconditioner, as given in Eq. (18). serves no benefits whatsoever. evell 
though the fluid speed is low in the viscous and the base flow regions. This is understandable because the preconditioner effects only the inviscid waves and the information in the viscous-dominated regions is only transmitted via diffusion processes which are ably handled by the implicit operator. Viscous and grid aspect effects can be included in the construction of preconditioner, see for example [16].

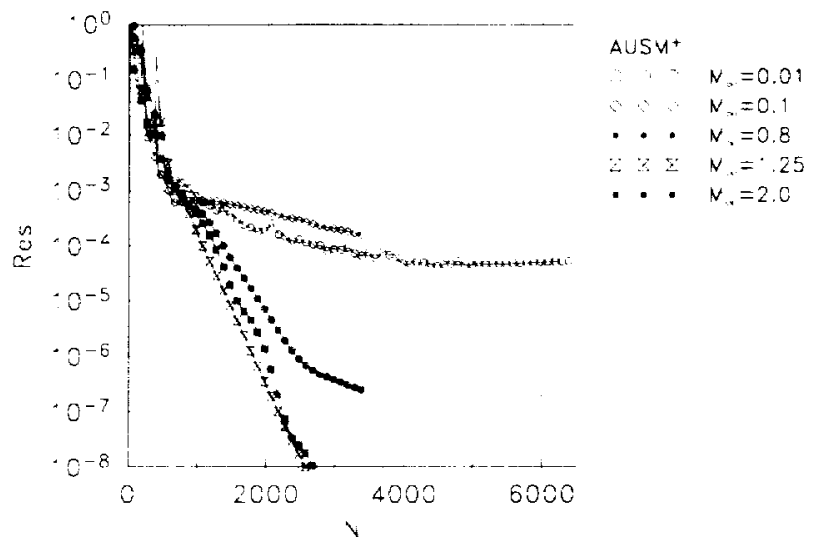

Fig. is ('onvergence history for the shut tle external tank problem.

In Fig. 5. we display the convergence history for various Wach numbers using $\mathrm{AISM}^{+}$. without using the preconditioner Г. The residuals for the low Mach-number cases stall after a drop of four orders of magnitude. These drops in many calculations. although not especially admirable, would have been acceptable. However. a close examination of the solution reveals that it is completely unacceptable. as shown in Fig. 6. It appears that there is a false boundary (exactly aligned with a grid line) at which information is unable to pass. This phenomenon is quite typical in the low Mach-number calculations using an unmodified compressible code, also seen in [12]. Hence. a measure of caution should be taken when reading the residual history for the low Mach-number solutions.

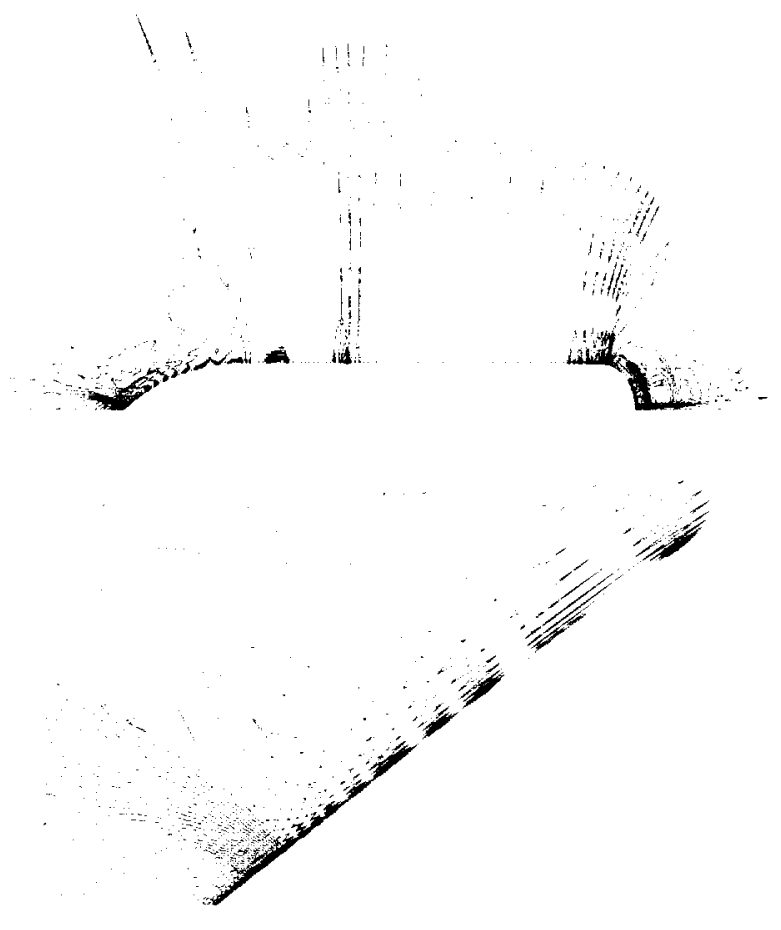

Fig. 6 Pressure contours for the shuttle external tank problem obtained at $\mathrm{N}=6400$ time steps for $M_{x}=0.01$, using the standard ACSM ${ }^{+}$. The Bottom picture shows a magnified view near the nose.

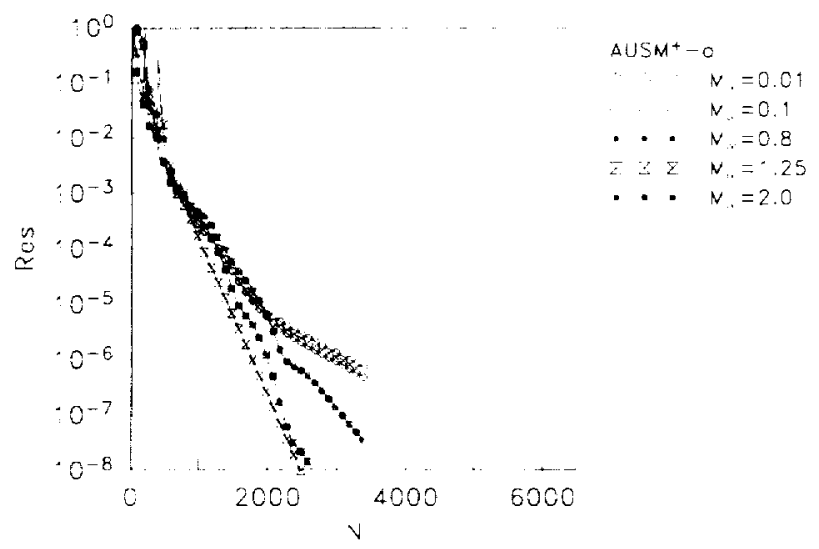

Fig. i ('onvergence history for the shuttle external tank problem obtained by the $\mathrm{ACSM}^{+}-$a scheme.

On the other hand, the convergence his- 
tories with the use of the numerical speed of sound display improvement over those without it. as shown in Fig. 7 . As noted carlier in Table 1 , it is necessary to invoke $\Gamma$ for the $M_{x}=0.01$ and 0.1 cases. The convergence rates for these two calculations nearly coincide with each other. indicating Machnumber independence.

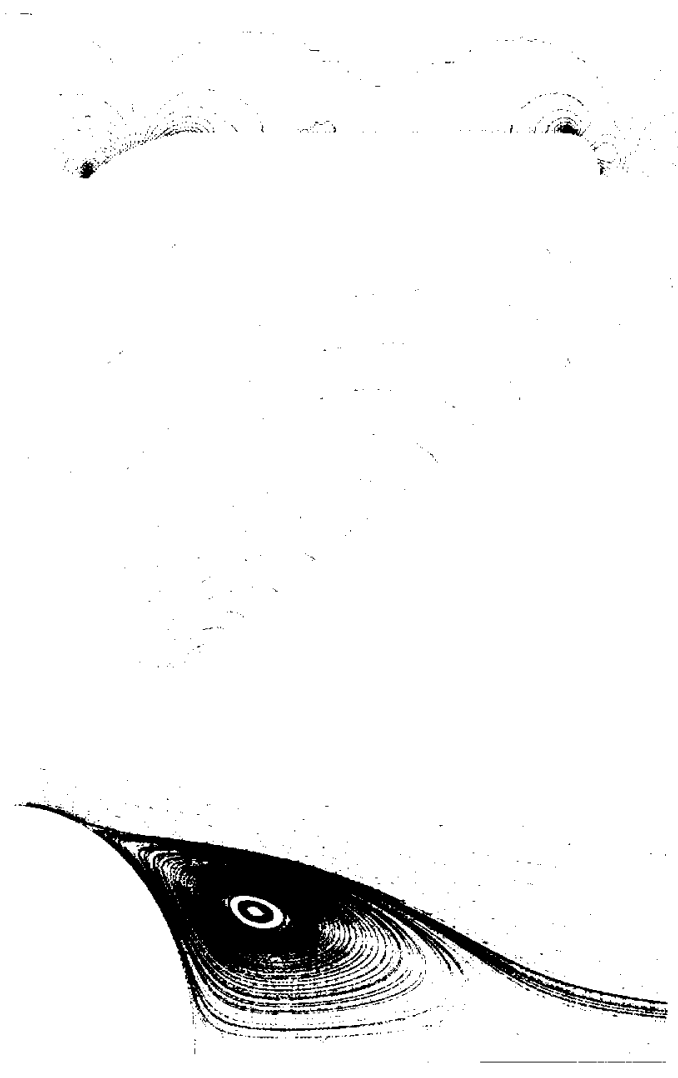

Fig. \& Pressure contours for the shuttle external tank problem obtained at $\mathrm{N}=1000$ time steps for $M_{\alpha}=0.01$. using USSM $^{+}-$a. The middle picture shows a magnified view near the nose and the bottom one depicts the separated zone behind the tank base.

In Fig. \&, we show the solution at $\mathrm{N}=1000$ steps at which the residual has been dropped to the level approximately equal to that shown in Fig. $6(\mathrm{~N}=6400)$. It is of interest noticing that the solution now is well behaved and is every bit as good as the solution at $N=3200$. Also the blow-up view near the surface reveals smooth profiles of pressure contours. unlike the standard $\mathrm{AISM}^{+}$which has been known to yield unwanted pressure oscillations in viscous layers along the transverse grid lines when the mesh aspect ratio is large and flow is essentially parallel to a grid line.* The separation region in the base of the tank is depicted by particle traces. indicating its size extending about one radius downstream.

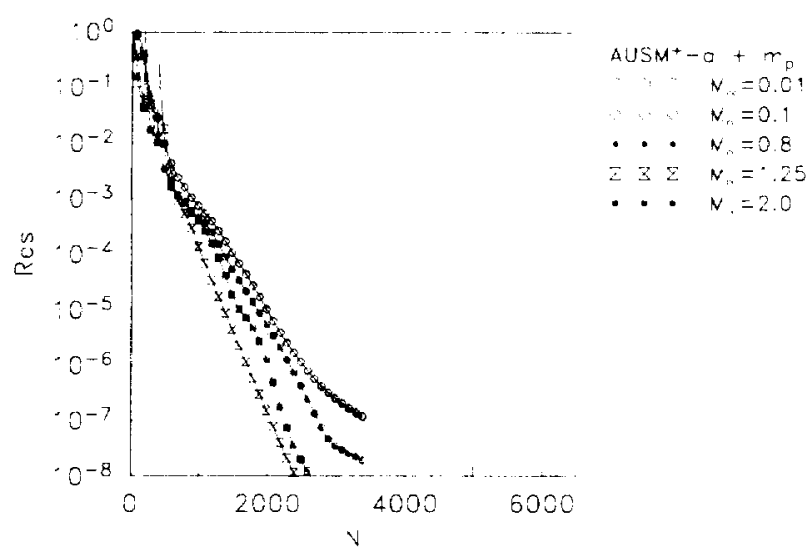

Fig. 9 Convergence history for the shut tle external tank problem obtained by the ACSM+ ap method.

Finally the effect of including the pressure diffusion term on the solution was investigated and the results are given in Fig. 9. Again. the preconditioner $I$ must be used for the low Mach-number cases and their convergence histories are essentially identical, becoming independent of Mach number as the Mach number lowers. The pressure contours are indistinguishable from those shown in Fig. 8 and are thus not included.

\footnotetext{
*However, the pressure distribution along the wall is smooth.
} 

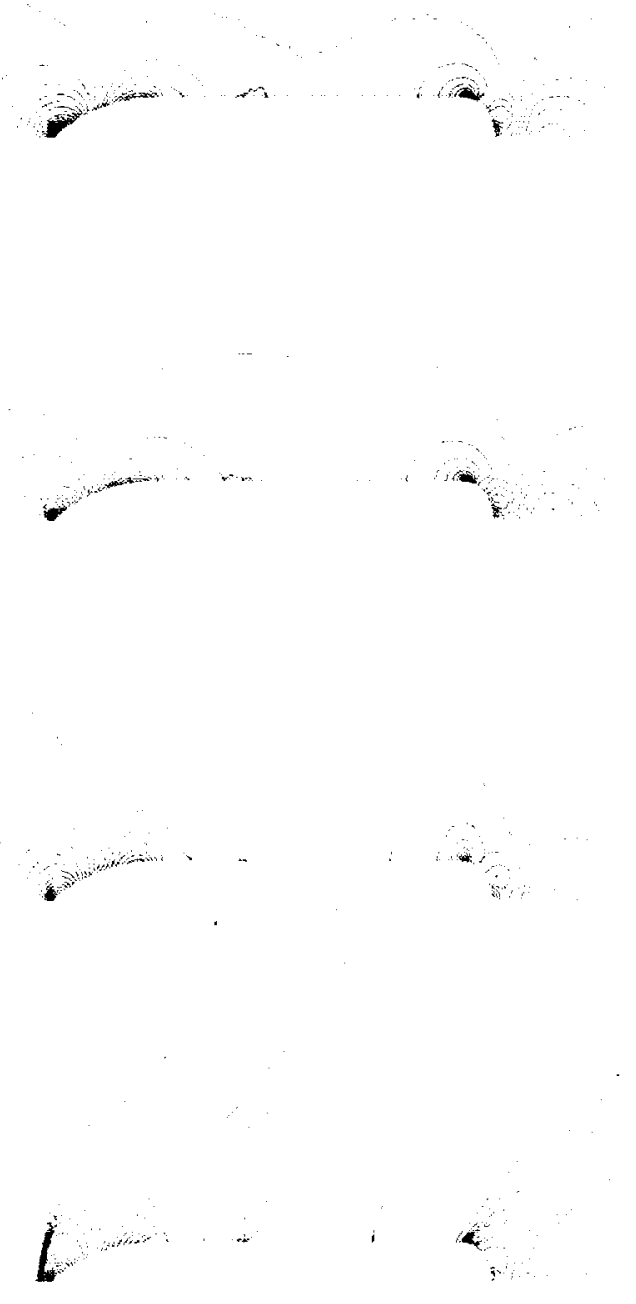

Fig. 10 Pressure contours at $H_{x}=$ $0.01 .0 .1,0 . \$ .1 .25$, and 2.0 (from top to bottom) for the shuttle external tank problem, showing accordingly different flow features.

The solutions obtained by using the
$\mathrm{AUSM}^{+}$- a for several $M_{\infty}$ values are given in Fig. 10 for comparison. They all appear physically correct and are numerically well behaved. No discenible differences were noticed between those by $\mathrm{AUSM}^{+}$-a and $\mathrm{AUSM}^{+}-$ap.

Comparing Figs. 5, 7 and 9, we see that the convergence rate is improved in the transonic ranges by simply using the numerical speed of sound alone. For low Mach number cases. $M_{x}=0.01,0.1$, another order of reduction can be obtained by including the pressure diffusion term. Also, the use of nutmerical speed of sound yields the convergence histories that are relatively insensitive to the flow speeds. Thus, the validity of the present method is confirmed and the goal of having a convergence rate that is more or less independent of the free-stream speed is achieved.

We now summarize major findings from the study of this problem: (1) The numerical speed of sound concept is an effective means of extending AUSM-type discretizations to solve low Mach number flows in an accurate and efficient manner. (2) Since the numerical speed of sound is reduced with the flow speed. the numerical dissipation changes accordingly: and a compatible implicit operator (one that includes the preconditioning matrix) must be used. (3) For moderate Mach numbers and beyond. it is not necessary to use $\Gamma$. (4) Incorporation of the numerical speed of sound, as described in steps (26)-(29). helps remove pressure oscillations in the viscous lavers.

\section{ONERA M6 Wing}

The next problem is the ONERA MG wing with the free stream conditions $M_{\infty}=0.84$, and $R \epsilon_{x}=18.2 \times 10^{6}$. 11nder various angles of attack. The computation domain consists of $269 \times 35 \times 67$ grid points. For this case. the preconditioning matrix $l$ was automatically turned off in the code since the controlling factor $3 M_{x}^{2}$ exceeds unity. However, the 
numerical speed of sound à was active with $M_{c o}=M_{x} / 2$. The pressure contours on the wing surfaces are displayed in Fig. 11, showing the well-known $\lambda$-shock pattern, thus appearance of two shocks roughly in two thirds of the wing span. The detailed comparison of surface pressure distributions are shown for two spanwise sections. Figs. 12 and 13 respectively for $44 \%$ and $65 \%$, for four angles of attack. The computed results are in very good agreement with the data [17]. especially in capturing of the shock locations.

The convergence histories are presented in Fig. 14 for two different angles of attack. They show a continuing decrease by about five orders of magnitude from the largest values. at nearly the same rates.

\section{Wingbody}

Turbulent flows over a wingbody configuration are calculated and their results are now discussed. The geometry is shown in Fig. 15. where the sting is included in the calculation. The computation domain is griclded using the chimera overset grid technique and entire grid is composed of seven grids. The flow conditions are: $M_{x}=0.8,0=2^{\circ}$. and $h_{\mathrm{x}}=0.167 \times 10^{6}$. Figure 16 depicts the pressure coefficients at various spanwise locations. The computed results are in excellent agreement with the measured data [18]. Moreover, the pressure coefficients along the body, shown in Fig. 17. exhibits a similar level of excellent agreement with the data. even in the wing root region where a sharp variation is encountered.

Finally, Fig. 18 displays a well-behaved convergence history reducing the residual error by more than five (i) orders of magnitude in 800 steps.

\section{Concluding Remarks}

In this paper we have introduced the notion of "mumerical speed of sound", which t urns out to be a very useful variable that can be employed to construct an upwind scheme to satisfy certain properties, most notably the exact capturing of contact and shock discontinuities for $1 \mathrm{D}$ problems. This numerical speed of sound is explicitly utilized in the construction of $\mathrm{AUSM}^{+}$and AUSMDV schemes. However, the idea can be inserted in the other upwind schemes. One example is in the Roe flux splitting where an averaged speed of sound, among several other areraged variables. is automatically required for the process. We have shown that other celebrated schemes, which in standard form exhibit intermediate points, can now be made to capture a slock exactly.

More importantly, the concept of numerical speed of sound, being meaningful only in the numerical sense. can be extended to effectively deal with flows at low speeds. The crux is that a scaling factor varying with speed (or Macl number) is introduced. As a result, the numerical dissipation is decreased with the flow speed. Hence the convergence rate is enhanced. not only at low speeds. but also at high speeds as well. Additionally. the solution accuracy is improved.

The effectiveness of implenenting the numerical speed of sound in the A CSMI ${ }^{+}$scheme has been demonstrated. Solutions of complex turbulent flows were obtained for complicated geometries. meshed with the overset grid technique. using the OVERFLOW code. We have presented convergence histories demonstrating significant improvement over the previous scheme. The pressure distributions are in excellent agreement with available data for the ONERA M6 wing and a wingbody configuration. further proving the reliability of the new schene, Als $\mathrm{MI}^{+}$a.

\section{Acknowledgments}

The first author thanks Pieter Buning of Langley Research ('enter and William (than 
of Ames Research (enter, who have provided consultation concerning the OVERFLOW code and its utility tools. This study was sponsored by the Turbomachinery and Combustion Technology project. managed by Kaz Civinskas of Glenn Research Center.

\section{References}

1. B. van Leer. "Flux-rector splitting for the Euler equations," Lecture Notes in Physics 170. 507 (1982).

2. M.-S. Liou and ('. J. Steffen, "A new flux splitting scheme." J. Comput. Phys. 107.23 (199:3).

3. M.-S. Liou, "A continuing search for a near-perfect numerical flux scheme. Part I: AlSM+" NASA TM 106.524 (1994): also $J$ Comput. Phys. 129, 361 (1996).

4. Y. Wada and M.-S. Liou. "An accurate and robust flux splitting scheme for shock and contact discontinuities," SIAM J.SCi. (omput. 18. 633) (1997).

5. B. Einfeldt. "On Godunov-type methods for gas dymanics." SIAM J. Numer. Anal. 25. $29+(1988)$.

6. D. Hänel. R. Schwane, and G. Seider, "On the Accuracy of upwind schemes for the solution of the Narier-Stokes equations." sth AIAA CFD Conference. AIAA Paper 8t110.5-C'P. 1987.

7. Y. II. ('hoi and ('. L. Merkle, "The Application of preconditioning in viscons flows." $J$. Comput. Phys. 105. 207 (1993).

8. B. van Leer. W. T. Lee and P. L. Roe. "('laaracteristic time stepping or local preconditioning of the Euler equations." AIAA paper 91-1552. 1991.

9. E. Turkel. V. N. Vatsa and R. Radespiel, "Preconditioning methods for lowspeed flows." AIAA Paper 96-2460, 1996.

10. J. M. Weiss and W. A. Smith, "Preconditioning applied to rariable and constant density time-accurate flows on unstructured meshes." AI 1 A Paper 94-2209. 1994.
11. J. M. Weiss, J. P. Maruszewski and W. A. Smith, "Implicit solution of the preconditioned Navier-Stokes equations using algebraic multigrid," ALAA J. 37, 29 (1999).

12. J. R. Edwards and M.-S. Liou, "Lowdiffusion flux-splitting methods for flows at all speeds." AIAA J. 36, 1610 (1998).

13. J. R. Edwards, R. K. Franklin, and M.S. Liou, "Low-diffusion flux-splitting methods for real fluid flows at all speeds." AIAA Paper 99-33227. 1999.

14. P. G. Buning et. al. "OVERFLOW user's manual, version 1.8f" Inpublished NASA Report. 1998.

15. P. R. Spalart and S. R. Allmaras, "A oneequation turbulence model for aerodynamic flows." AIAA Paper 92-0439. 1992.

16. P. E. O. Buelow, S. Venkateswaran, and C. I. Merkle. "Grid aspect ratio effects on the convergence of upwind schemes," AIAA Paper 95-0565. 1995.

17. V. Schmitt and F. ('harpin. Appendix B1. A(ARD-AR-138, 1984.

18. D.A. Treadgold. A.F. Jones, and K.H. Wilson, Appendix BH. AGARD-AR138. 1984 .

19. B. Koren. "Ipwind schemes, multigrid and defect correction for the steady NavierStokes equations." Lectur Notes in Physics 323. $314(1989)$

\section{$\underline{\text { Appendix A }}$}

In this section we give a fornal derivation of the function $\mathcal{D}$ that appears in the pressure diffusion term in mass flux. We begin with the AUSMDV flux written in terms of the numerical speed of sound.

$$
\begin{array}{r}
\dot{m}_{1 / 2}=\dot{a}_{1 / 2}\left[\rho_{j} \mathcal{M}_{(1)}^{+}\left(M_{j}\right)+\rho_{j+1} \mathcal{M}_{(1)}^{-}\left(M_{j+1}\right)\right. \\
+\rho_{j \omega^{+}}\left(\mathcal{M}_{(4, j)}^{+}\left(M_{j}\right)-\mathcal{M}_{(1)}^{+}\left(M_{j}\right)\right) \\
\left.+\rho_{j+1} \omega^{-}\left(\mathcal{M}_{(4,3)}^{-}\left(M_{j+1}\right)-\mathcal{M}_{(1)}^{-}\left(M_{j+1}\right)\right)\right] .
\end{array}
$$

where

$$
\rho_{j \omega^{+}}=\frac{p_{j}}{D}, \quad \rho_{j+1 \omega^{-}}=\frac{p_{j+1}}{D} .
$$


These can be rewritten as

$$
\begin{aligned}
\rho_{j} \omega^{+} & =\frac{1}{2 D}\left(\sum p-\Delta p\right), \\
\rho_{j+1} \omega^{-} & =\frac{1}{2 D}\left(\sum p+\Delta p\right),
\end{aligned}
$$

together with

$$
\sum p=p_{j}+p_{i+1}, \quad \Delta p=p_{j+1}-p_{j} .
$$

Noticing that $D$ has the dimension of $p / \rho$. Substituting these formulas into Eq. (1), we denote the coefficient for $\Delta p$ term by

$$
\kappa_{p}=-\frac{\grave{a}_{1 / 2}}{2 D} \Delta \mathcal{M}
$$

where we have $\triangle . \mathcal{M}$ given in $\mathrm{Eq}$. (33). As $M_{j}, M_{j+1} \rightarrow 0$. we get

$$
\Delta M=\left(\frac{1}{2}+3\right)
$$

Hence, if $D=O(1)$. then

$$
K_{p}=O\left(u_{1 / 2}\right), \quad \text { as } u_{1 / 2} \ll 1 \text {. }
$$

That is. the pressure diffusion term diminishes with the flow speed and it clearly is insufficient to provide adequate contribution of pressure to the mass flux at low speeds. This can be remedied by properly rescaling $K_{p}$, as

$$
\left.K_{p}^{\prime}=\frac{\grave{a}_{1 / 2}}{2 M_{*}^{2} D}\right\lrcorner \mathcal{M}
$$

As a result, we should have defined $\omega^{ \pm}$in the following way,

$$
\begin{aligned}
\rho_{, \omega^{+}} & =\frac{1}{2 D}\left(\sum p-\frac{\Delta p}{M_{*}^{2}}\right) . \\
\rho_{i+1} \omega^{-} & =\frac{1}{2 D}\left(\sum p+\frac{\Delta p}{M_{*}^{2}}\right) .
\end{aligned}
$$

What is left is to determine the expression of $D$. First. we remark that the condition for capturing a stationary contact discontinuity requires

$$
\rho_{j \omega^{+}}=\rho_{j+1} \omega^{-}
$$

Monotonicity constraints on the "+ " and ".." split Mach numbers in Eq. (1) implies conditions on $\omega^{ \pm}$(see [4] for example).

$$
0 \leq \omega^{+}, \omega^{-} \leq \frac{2}{1+13}
$$

A more stringent condition fulfilling the above inequalities may be

$$
0 \leq \omega^{+}+\omega^{-}=\omega \leq \frac{2}{1+13} .
$$

This gives the relation

$$
\begin{aligned}
\mathcal{D}=M_{\times}^{2} D=\frac{1}{\omega \rho_{j} \rho_{j+1}}[ & M_{*}^{2}\left(p_{, j}+p_{j+1}\right)\left(\rho_{j}+\rho_{j+1}\right) \\
& \left.-\left(p_{j}-p_{j+1}\right)\left(\rho_{j}-\rho_{j+1}\right)\right] .
\end{aligned}
$$

This completes the derivation of $\mathcal{D}$. 


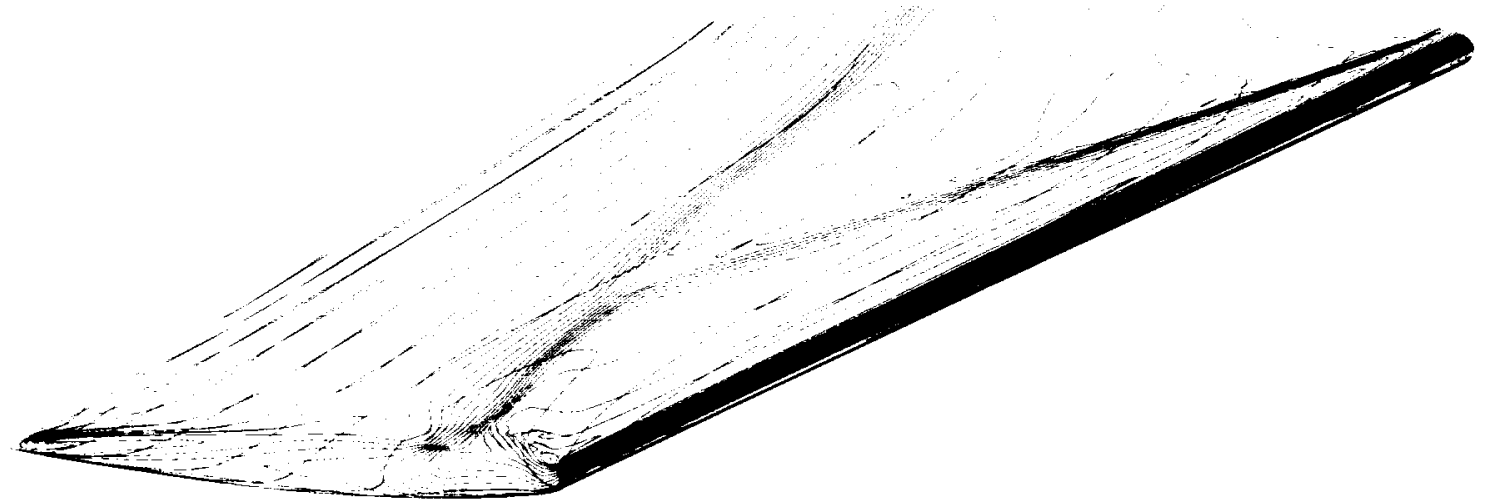

Fig. 11 Pressure contours on the ONERA $M 6$ wing at $\alpha=3^{\circ} . M_{x}=0.84$, and $\operatorname{Re} e_{x}=18.2 \times 10^{6}$, slowing the $\lambda$-shock pattern near the wing tip.
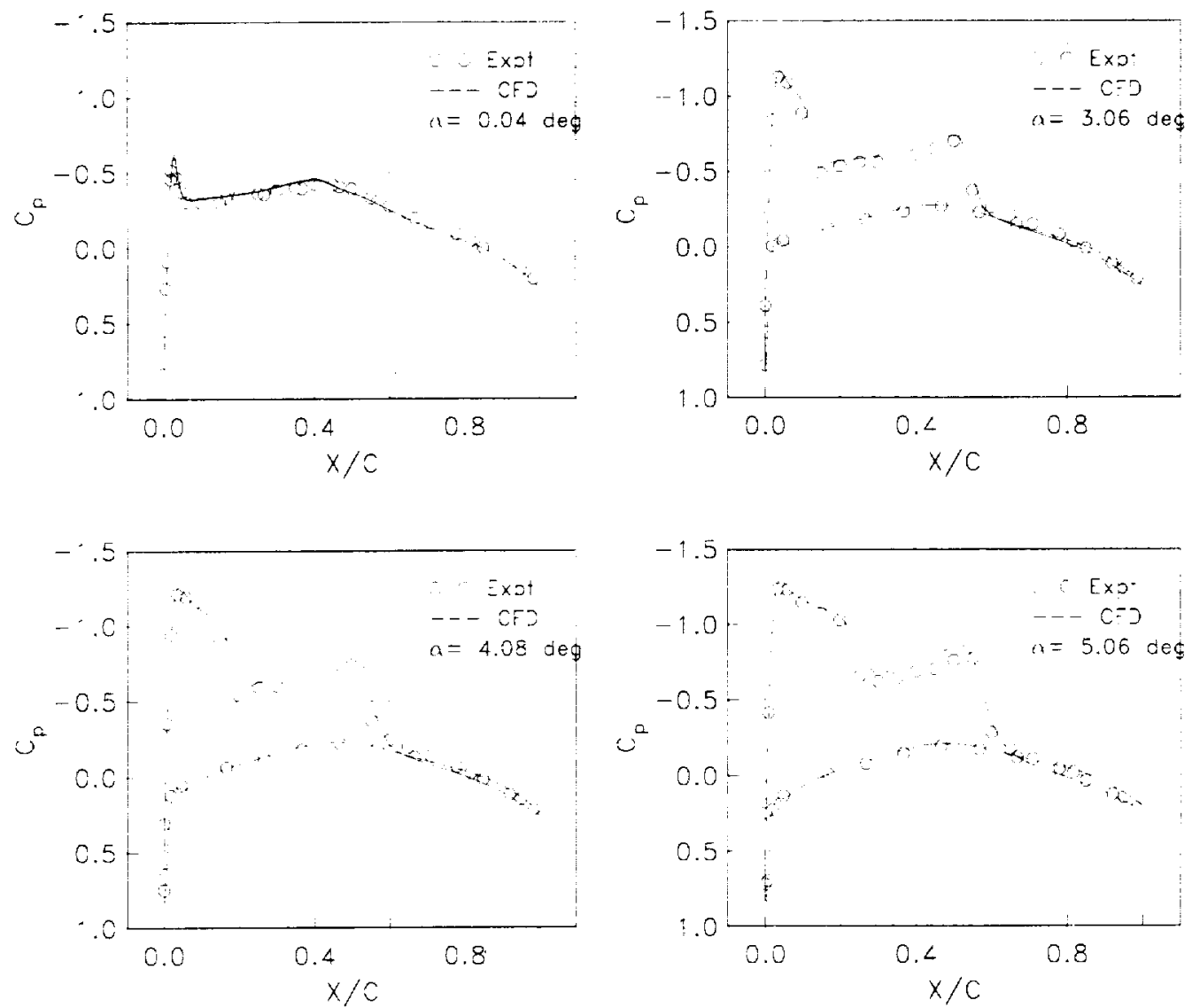

Fig. 12 Pressure distribution at the spanwise section. $44 \%$ of the ONERA M6 wing for various angles of attack, $M_{x}=0$. S. $R \mathrm{f}_{\mathrm{x}}=18.2 \times 10^{6}$. 

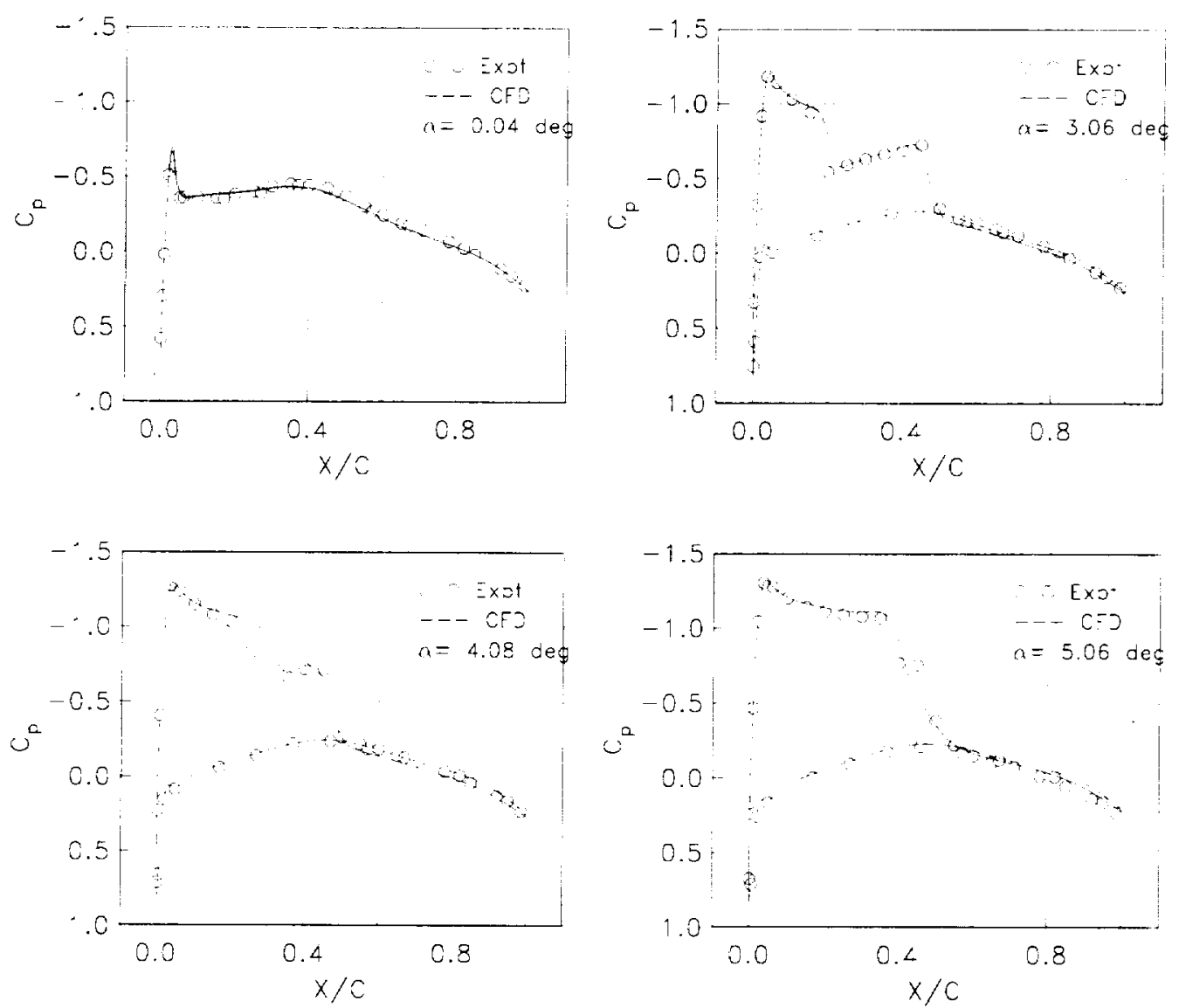

Fig. 13 Pressure distribution at the spanwise section. $65 \%$, of the ONERA M6 wing for varions angles of attack. $M_{x}=0.84 . R c_{x}=18.2 \times 10^{6}$.
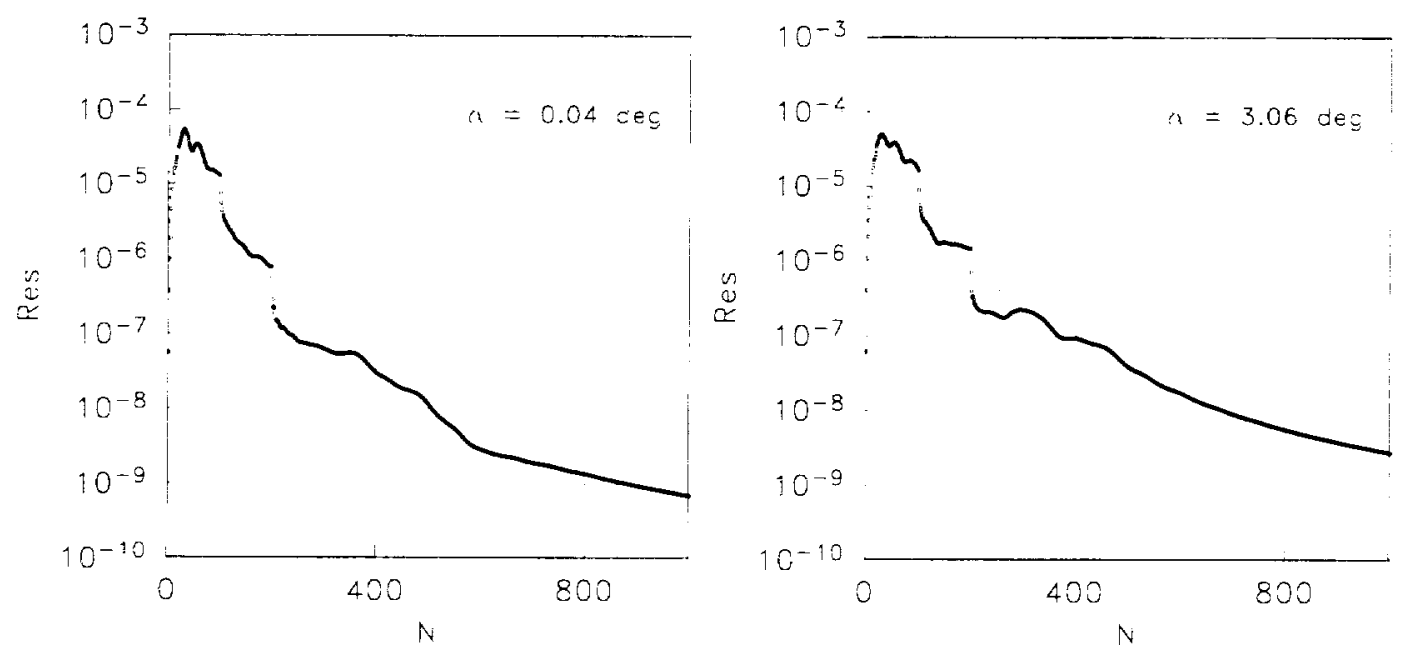

Fig. It (onvergence history for the ONERA MG wing problem at two angles of attack. $M_{x}=$ 0.8. $\operatorname{Re}_{x}=18.2 \times 10^{6}$. 


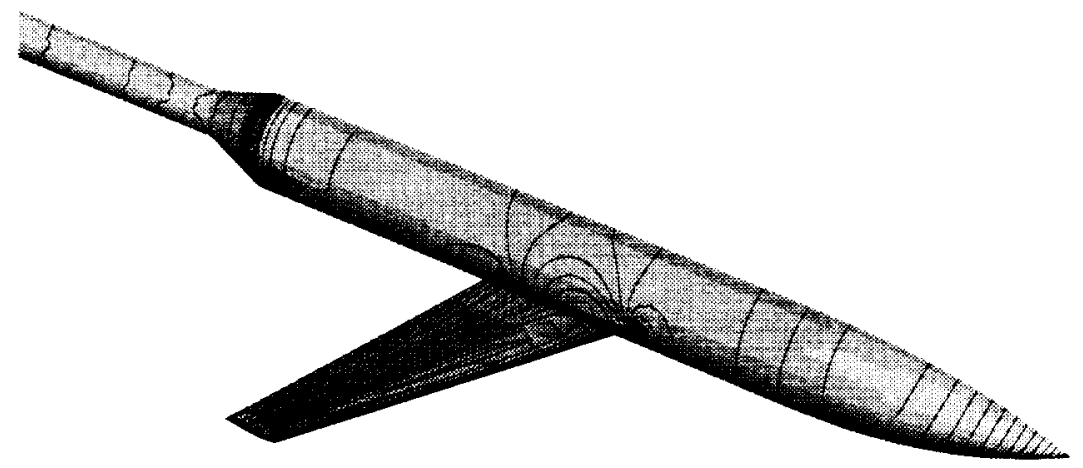

Fig. 15 Geometry of the wingbody probleni.
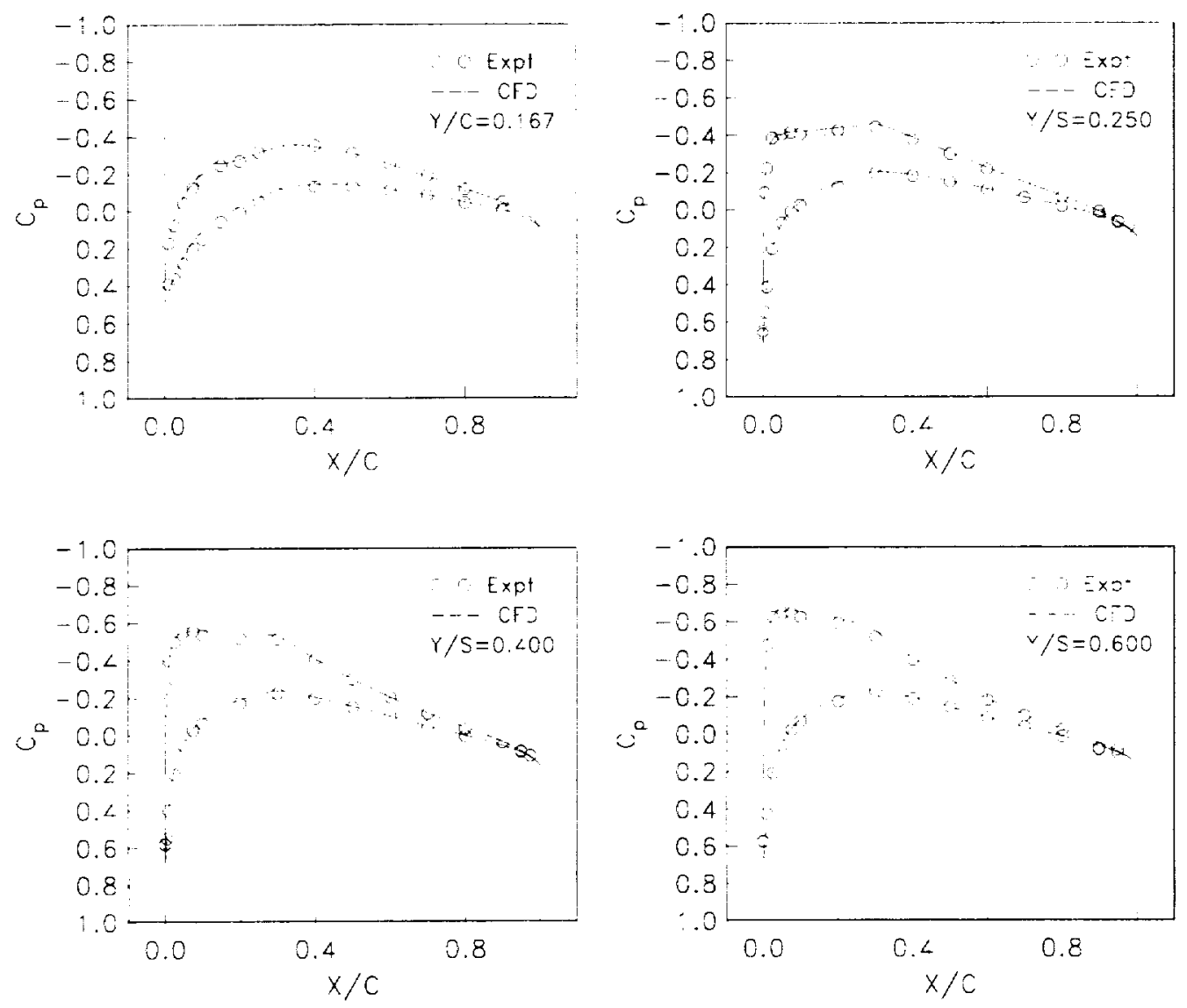

Fig. 16 Pressure distribution at various sections of the wing. $M_{x}=0.8, a=2 . R 6 \times=0.167 \times 10^{6}$. 

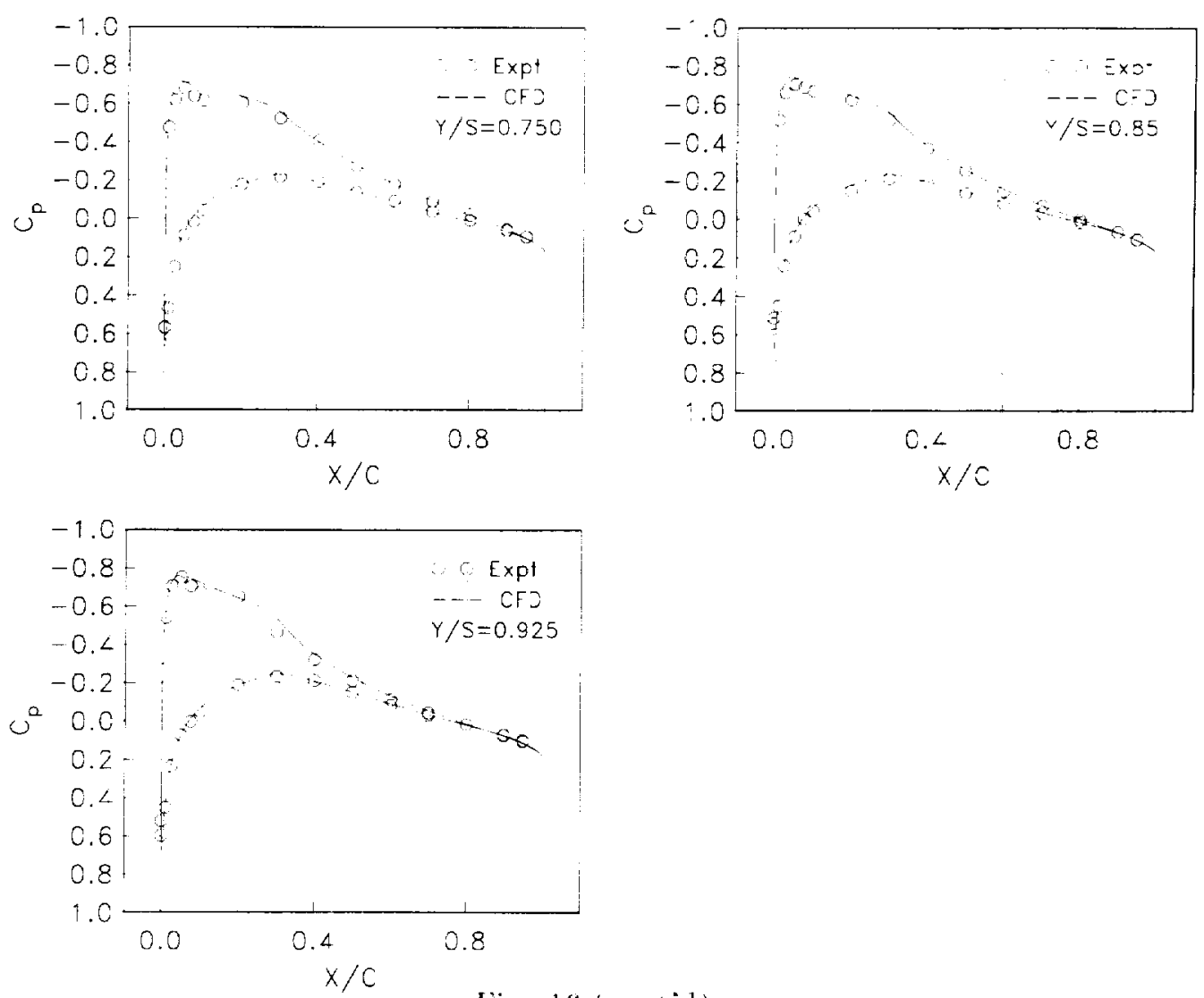

Fig. 16 ( cont $\left.^{\circ} \mathrm{d}\right)$
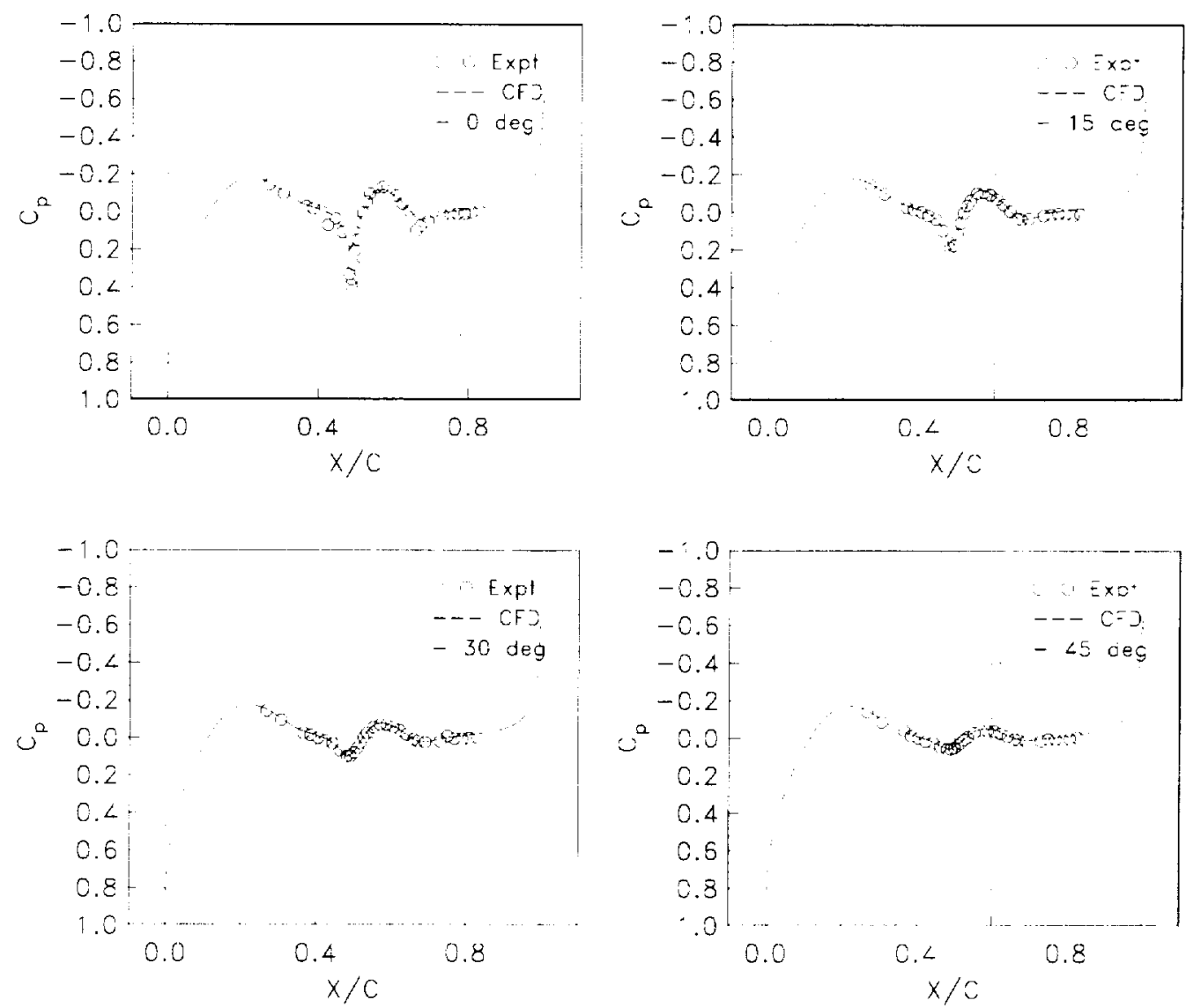

Fig. 17 Pressure distribution at angles along the body, $M_{x}=0.8, n=2^{\prime \prime}$. Re $\mathrm{x}_{x}=0.166^{-} \times 10^{6}$. 

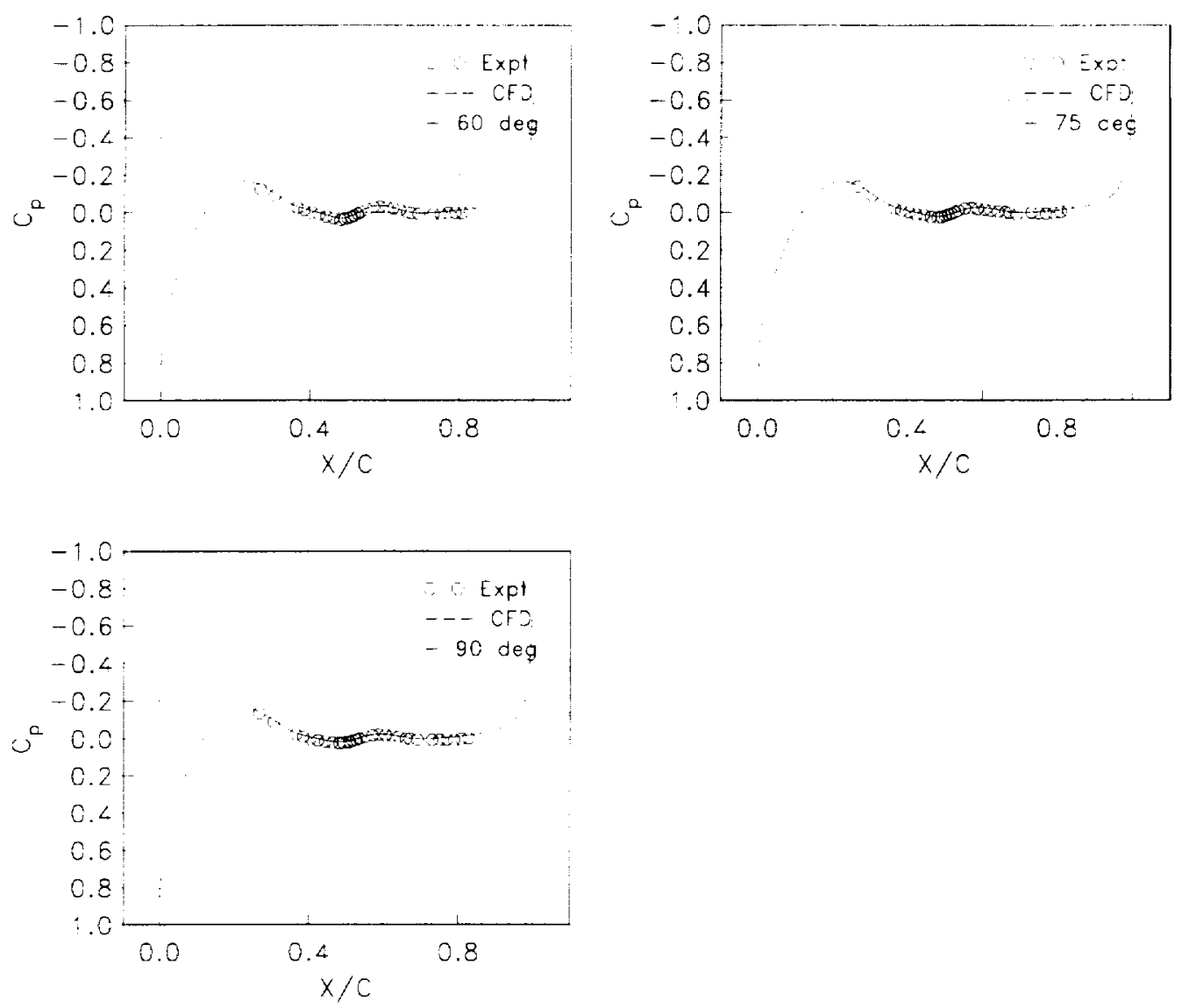

Fig. $17\left(\right.$ cont $\left.^{*} \mathrm{~d}\right)$

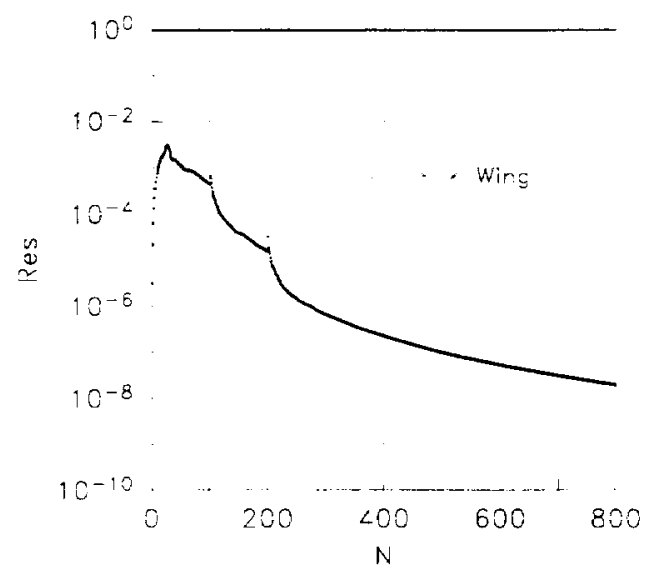

Fig. 1s convergence history for the wingbody problem. 



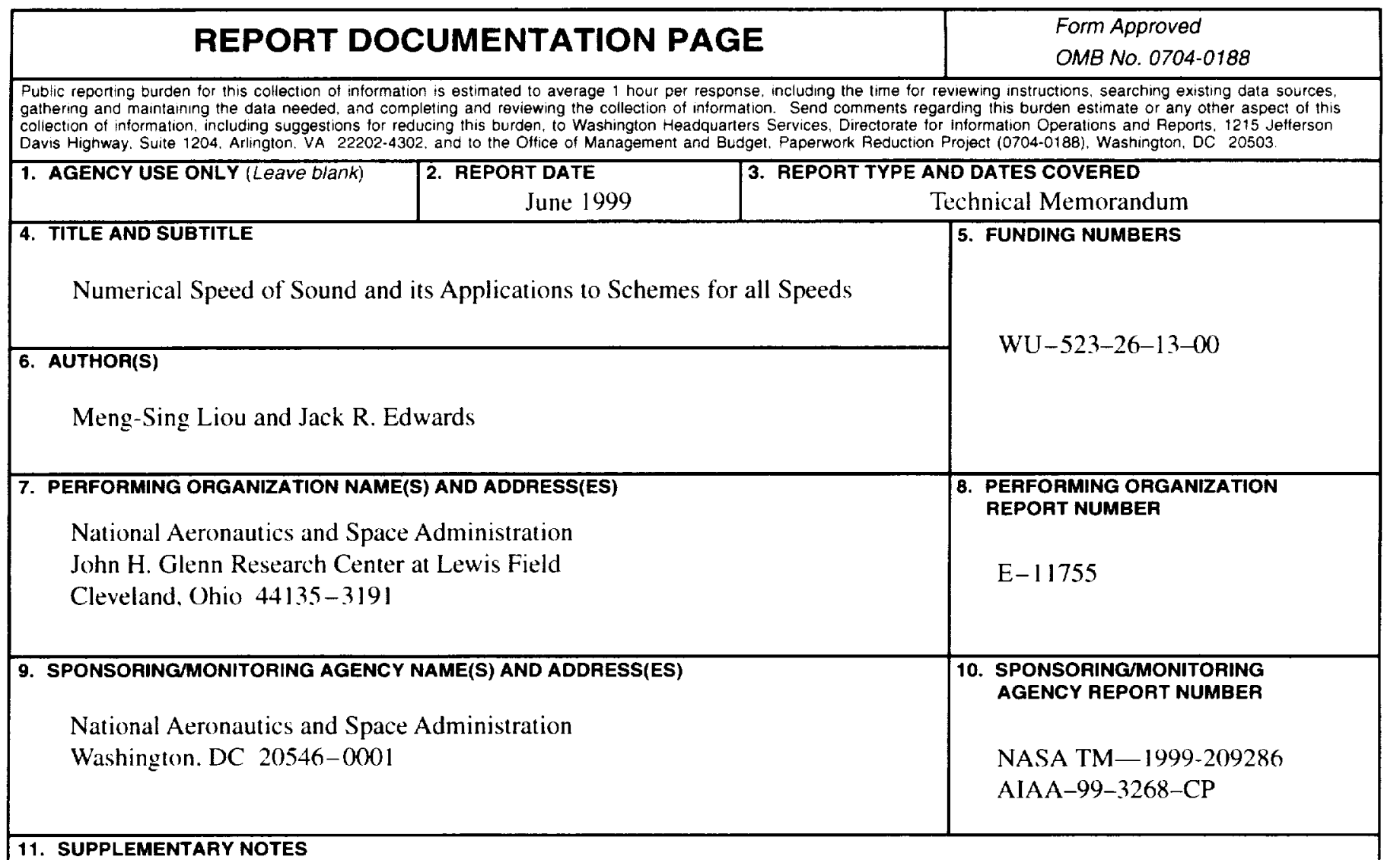

Prepared for the 14th Computational Fluid Dynamics Conference sponsored by the American Institute of Aeronautics and Astronautics, Norfolk. Virginia, June 28-July 1. 1999. Meng-Sing Liou, NASA Glenn Research Center. and Jack R. Edwards. North Carolina State University, Raleigh, North Carolina 27695. Responsible person, Meng-Sing Liou, organization code 5880. (216) 433-5855.

12a. DISTRIBUTION/AVAILABILITY STATEMENT 12b. DISTRIBUTION CODE

Unclassified - Unlimited

Subject Categories: 02 and $64 \quad$ Distribution: Nonstandard

This publication is available from the NASA Center for AeroSpace Information. (301) 621-0390.

13. ABSTRACT (Maximum 200 words)

The concept of "numerical speed of sound" is proposed in the construction of numerical flux. It is shown that this variable is responsible for the accurate resolution of discontinuities, such as contacts and shocks. Moreover, this concept can be readily extended to deal with low speed and multiphase flows. As a result, the numerical dissipation for low speed flows is scaled with the local fluid speed, rather than the sound speed. Hence, the accuracy is enhanced, the correct solution recovered, and the convergence rate improved. We also emphasize the role of mass flux and analyze the behavior of this tlux. Study of mass flux is important because the numerical diffusivity introduced in it can be identified. In addition, it is the term common to all conservation equations. We show calculated results for a wide variety of flows to validate the effectiveness of using the numerical speed of sound concept in constructing the numerical flux. We especially aim at achieving these two goals: (1) improving accuracy and (2) gaining convergence rates for all speed ranges. We find that while the performance at high speed range is maintained, the flux now has the capability of performing well even with the low speed flows. Thanks to the new numerical speed of sound, the convergence is even enhanced for the flows outside of the low speed range. To realize the usefulness of the proposed method in engineering problems. we have also performed calculations for complex 3D turbulent flows and the results are in excellent agreement with data.

\begin{tabular}{|c|c|c|c|}
\hline \multicolumn{3}{|l|}{ 14. SUBJECT TERMS } & $\begin{array}{c}\text { 15. NUMBER OF PAGES } \\
26 \\
\end{array}$ \\
\hline
\end{tabular}

NSN 7540-01-280-5500 